\title{
Experimental Study on the Characteristics of Granular Materials' Flows and Fractures under Uniaxial Compression
}

\author{
Futian Zhao, ${ }^{1,2,3}$ Jun Liu $\mathbb{D}^{1},{ }^{1,2,3}$ Zhimin Xiao, ${ }^{1,2,3}$ Mingqing Liu, ${ }^{1,2,3}$ Yue Wang, ${ }^{1,2,3}$ \\ Chen Ou, ${ }^{1,2,3}$ and Mengyang Zhen ${ }^{1,2,3}$ \\ ${ }^{1}$ Key Laboratory of Ministry of Education for Geomechanics and Embankment Engineering, Hohai University, \\ Nanjing 210098, China \\ ${ }^{2}$ Institute of Engineering Safety and Disaster Prevention, Hohai University, Nanjing 210098, China \\ ${ }^{3}$ College of Civil and Transportation Engineering, Hohai University, Nanjing 210098, China
}

Correspondence should be addressed to Jun Liu; ljun8@263.net

Received 15 July 2020; Revised 26 October 2020; Accepted 10 November 2020; Published 7 December 2020

Academic Editor: Chunshun Zhang

Copyright (C) 2020 Futian Zhao et al. This is an open access article distributed under the Creative Commons Attribution License, which permits unrestricted use, distribution, and reproduction in any medium, provided the original work is properly cited.

\begin{abstract}
The mechanical behaviors of granular materials have high complexity. Since the mechanical behaviors of granular materials are difficult to describe theoretically, in this paper, the flow and fracture characteristics of granular materials were discussed by the experimental method. An experimental method was firstly proposed to identify the mechanical behaviors of granular materials under extra loading. An experimental device, which can be used to monitor force and displacement between particles, was invented. The uniaxial compression experiments of granular particles with different materials and grades were carried out, and the overall and local force between particles was obtained and investigated. Due to the flow of particles, the overall force rises in a fluctuating manner and the local force is not distributed evenly. Then, the particle fragments were sieved layer by layer. It was confirmed that the particles and loading strength have great influences on fractures of granular materials
\end{abstract}

\section{Introduction}

Granular materials consist of discrete particles and surrounding voids. The mechanical behaviors of granular materials are involved in many engineering fields, such as mining engineering, mineral processing engineering, blasting engineering, hydraulic engineering, railway engineering, and so on. The flows and fractures of granular materials under external loading are very common and play important roles in these related engineering fields, such as ore crushing, mechanical behaviors of rockfill dam, the flows and fractures of railroad slag, and so on.

The flows and fractures of granular materials are very complicated because the undisturbed granular materials resemble solid and the disturbed granular materials exhibit fluid characteristics. Therefore, the mechanical behaviors of granular materials are different from solids and fluids, which determines the complexity of its mechanical behaviors $[1,2]$. During flow process, the collision between particles or particles and boundary would cause particle breakage, the broken particles would be incorporated into the particle system and continue to flow as part of the particle system, and further fractures would occur [3-11]. However, there are many factors [12-20] that affect the flows and fractures of granular materials, such as particles' strength, size, shape, gradation, and loading rate. Due to the complexity of mechanical properties of granular materials, great efforts have been devoted to study the flows and fractures of granular materials in the past decades, and the main research methods are experiment and numerical simulation.

Experimental research is divided into the single particle test and the particle system test. The purpose of single particle test is to establish the relationship between the strength, shape, and size of particle and the degree of particles' fractures under different loading conditions and to identify the influencing factors and fracture mechanism. Gorham and Salman and Fu et al. [21, 22] analyzed the failure patterns under impact loading for single spherical 
particle made of glass, polymethyl methacrylate, and marble powder by impact tests. The results indicated that dynamic effects of particle under impact were clearly important, even at comparatively low-impact velocities. The high-velocity failure mode is not reproducible by any quasistatic test and is a consequence of the very high loading rate as the impact stresses reach very high values. Antonyuk et al. [23-25] studied the breakage behaviors of single $\gamma-\mathrm{Al}_{2} \mathrm{O}_{3}$ spherical particle by compression and impact tests. The breakage mechanisms of granule during elastic, elastic-plastic, and plastic deformation are explained. Chau et al. [26] proposed a new stress analysis method for isotropic elastic spheres compressed between two flat rigid platens. In this method, the size of the contact zone can be estimated quite accurately in terms of the granule size, the dynamic hardness, and the impact energy. The impact energy for fragmentation increases monotonically with the size and strength of the granules. By comparing the static and dynamic tests, an empirical formula is proposed to estimate the impact energy needed to break a granule if the static energy for fragmentation is known. Schubert et al. [27] studied the size distribution of fragments from a concrete granule failure under impact loading. A formula was proposed by Astrom and Herrmann [28] to describe the fragment size distribution by means of a statistic method. Olivier et al. [29] analyzed the size effects in single grain fragmentation by compression tests and two fracture modes were introduced, i.e., volume fracture and contact fracture. Jansen and Stoyan [30] verified the validity of the Weibull failure model for single brittle granule by compression tests.

Compared to the single particle test, the loads and interaction between the particles in the particle system test are often complicated, considering not only compression and shear stress, but also the sliding friction and rolling friction between particles. Moreover, the particle breakage is still an important factor affecting the mechanical behavior of granular material.

The uniaxial compression test of a sample consisting of the same particles shows that the yield strength of granular material is related to the average tensile strength of the particles [31]. Lade and Yamamuro [32,33] pointed out that the high confining pressures cause large amounts of particle crushing, which in turn cause the development of large positive pore pressures. This led to the effective stress path to move rapidly toward the effective stress failure envelope. The Mohr-Coulomb strength, effective stress, and angle of secant friction are approximately the same in compression and tension. Vallejo et al. [34] conducted particle compression crushing tests using the same size glass beads and simulated the experimental process by the discrete element methods, the results show that the granular material forms a new system, and the particle size distribution in the new system presents a natural fractal feature. The above studies analyzed the mechanical behavior of granular material composed of the same size particles; however, the particle size distribution is one of the important parameters that describe the composition of granular material, which directly affects the constitutive relation of granular material [35]. Grine et al. [36] conducted shear fracture experiments of carbonate sand; as the proportion of silica sand and cement increases, the ability of the particles to resist crushing increases, and when the silica sand and cement are added to a certain proportion, the shear strength of particles reaches a maximum value. The compression experiments of three different granular materials under high stress condition were performed by Hagerty [37]; particle crushing is influenced by initial void ratio, particle size, particle angularity, and particle material composition. Three phases of compression behavior were identified: volume decrease as a result of particle rearrangement at low stresses; more intense compression as particles crush and are rearranged extensively under higher stresses; and pseudo elastic behavior characterized by very high values of constrained modulus, as the number of contacts between fractured particles increases greatly at very high stresses.

Due to the large number and fluidity of particles, it is difficult to measure the contact force between particles by experimental methods; with the development of computers, numerical simulation methods have become an effective way to study the mechanical behavior of granular material. The discrete element method (DEM) was proposed by Cundall in 1971; DEM establishes an explicit equation group based on the motion equation of each unit; according to Newton's second law and constitutive relation, the equation group can be solved by time-step iteration. DEM is suitable for dealing with large deformation, large displacement, and dynamic problems, as DEM does not necessarily need the particle motion to satisfy the continuous displacement [18, 38-46]. However, the contact force model in DEM simulation is based on assumptions and simplifications, and the calculation results are all approximate values. The parameters of contact force model are difficult to determine, such as stiffness, time-step, and so on. If the values of parameters are unreasonable, it may cause a large difference between the simulation results and experimental results.

The mechanical properties of granular material have high complexity compared to the continuous material, and the existing experimental and numerical methods have some limitations. The current research studies are mainly focused on the influence of particle breakage on the overall mechanical behavior of granular material, lacking the analysis to the local mechanical behavior of granular material. Therefore, systematic experimental studies on granular materials with different sizes and gradations are important for identifying the flow and fragmentation mechanism of granular materials.

In this paper, a systematic method was proposed to measure the mechanical behavior of granular material. A mold for making spherical particles was produced, which can produce different sized particles. The experimental fixture was improved, and through the fixture, the mechanical experiments of granular material can be performed on conventional pressers. Space for sensors was reserved on the fixture to monitor the local and overall loads of particles, and the stress-strain relationship of the particles can be obtained during loading. After loading, the particle size distribution can be counted by the layer-layer sieve test to analyze the damage characteristics of particles. 
In the following sections, the experimental method and equipment were first introduced. Then, the results and some phenomena were also illustrated. Finally, the main conclusions from the study are drawn.

\section{Experiment Method}

In this experiment, a number of cement mortar particles of diameter 3,4 , and $5 \mathrm{~cm}$ were made by the designed mold, and a number of cement particles of diameter $0.6-2.2 \mathrm{~cm}$ were made by hand. Then, the particles were grouped, and the crushing experiments were carried out by groups. The experimental fixture was improved, and the mechanical experiments of granular material can be performed on conventional presses. During loading, the local and overall loads and displacement can be measured by the pressure sensors and electronic displacement meters. After loading, the fixture is divided into three layers according to the height, and the sieving experiments are performed on each layer to obtain the size distribution of particles.

2.1. Experimental Specimen. Experimental specimens are of two types: (1) cement mortar particles and (2) cement particles. Cement mortar particles are made of cement, sand, and water in a certain ratio (see Figure 1). The cement used is Portland cement with strength of $32.5 \mathrm{MPa}$, and the fineness modulus of sand is between 2.3 and 3.0. The diameters of particles are 3,4 , and $5 \mathrm{~cm}$, the shape of particles is regular, and size error is small. The shape of particles made by hand is similar to sphere, and the diameter ranges from $0.6 \mathrm{~cm}$ to $2.2 \mathrm{~cm}$.

2.2. Experimental Setup. In this work, the loading was performed by the electrohydraulic servo machine, and the pressure sensor and electronic displacement meter are used to measure the local and overall loads and displacement. The monitored signal is saved by a computer through a dynamic resistance strain gauge (see Figure 2).

An experimental device as a container for granular material was developed, and the device has four parts: fixture, upper bearing plate, lower bearing plate, and pin (see Figure 3).

The fixture is a cylindrical barrel, and the upper inner wall diameter is slightly larger than the lower. The pressure sensor can be placed inside the fixture, and the data line can be led through the hole in the lower part of the fixture. The lower bearing plate is a round steel plate with two holes, and the holes are symmetrical about the axis of the bearing plate. The pins are two solid steel cylinders, which are placed into the holes of the lower bearing plate. The surfaces of pins are in the same plane as the surface of the lower bearing plate, and the other sides of pins are connected to the pressure sensors. The upper bearing plate has two parts: a bolt and a round steel plate with a central threaded bore, and the two parts can be easily combined and split.
2.3. Experimental Process. Seven groups of experiments are performed (see Table 1). For each group, the size and number of particles are the same, but the loading strength is different.

The connection of the experimental instruments is shown in Figure 4. The pressure sensors and the electronic displacement meters are connected with the dynamic resistance strain meter, the pressure sensor on the upper bearing plate is used to measure the overall load, and the electronic displacement meter is used to measure the overall displacement. During loading, the particles flow under the external load, and when the particles at the bottom of the fixture are in contact with the pins on the lower bearing plate, the local pressure can be monitored by the pressure sensors, which are connected with the pins. After loading, the height of the whole particles is measured; according to the height, the particles are divided into three layers, and the fragments in each layer are sieved separately; finally, the size distribution of particles could be obtained.

\section{Experimental Result and Analysis}

Results of the experimental study are given in this section; fragment distribution and layered fracture characteristics will be investigated.

3.1. Overall Load. For the particles with different sizes under the same loading, the overall load-time curves are shown in Figure 5. The overall load change process of cement mortar particles can be divided into three stages. (1) Slow rising stage: in the initial stage of loading, the increase of overall load is relatively slow. Before the loading, the particles are in a state of natural accumulation and the porosity is large; as the applied load increases, the particles begin to flow and rearrange. (2) Fluctuating rising stage: with the increase of applied loading, the particle rearrangement is completed, and then the overall load change rate increases rapidly. When the applied load exceeds the particle crushing strength, the particles break and the broken particle fragments gradually fill into the voids of the particle system, resulting in a rapid increase of the overall displacement. At the same time, secondary flow and rearrangement occur in the new particle system formed by the broken particles. As the applied loading rate is less than the displacement increasing rate, an unloading process occurs. When the bearing plate is in close contact with the particles again, the overall load continues to increase. This loading-unloadingreloading process causes the overall load of the particles to rise in the form of fluctuation. (3) Rapid rising stage: as the particles continue to break, all the voids are filled, the particles become a system similar to the continuous medium, and the overall load no longer fluctuates and increases at a steady rate to the end.

For the cement particles, the overall load increase rate is relatively stable during the loading process and there is no obvious fluctuation in the force time-history curve. The main reason is that the particle system composed of small cement spheres has good gradation, high density, and low porosity. 


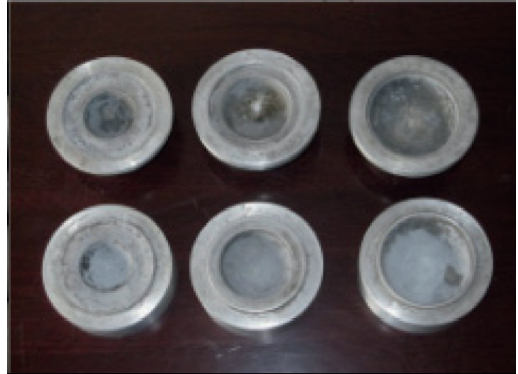

(a)

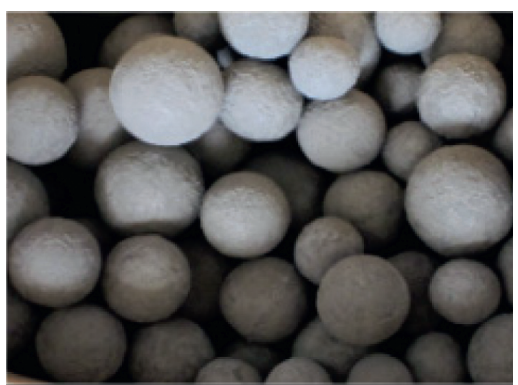

(b)

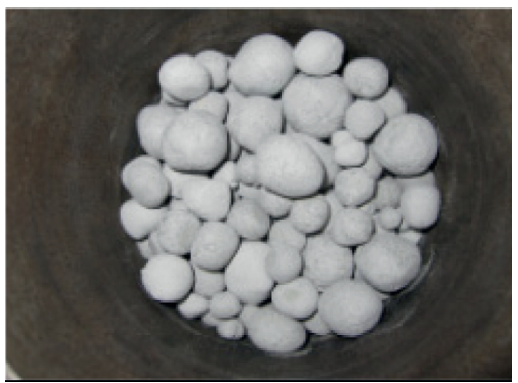

(c)

FIgURE 1: Experimental specimen: (a) the mold; (b) the cement mortar particles; (c) the cement particles.

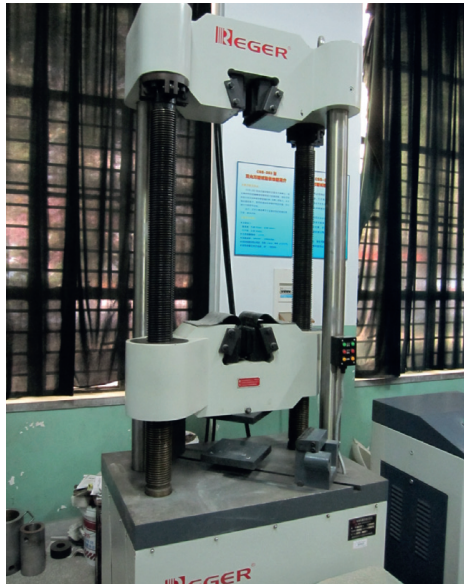

(a)

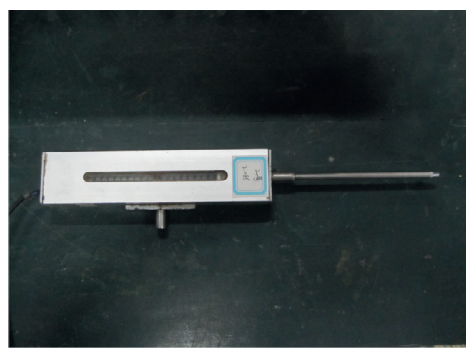

(c)

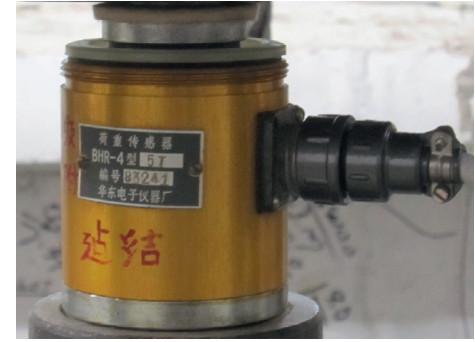

(b)

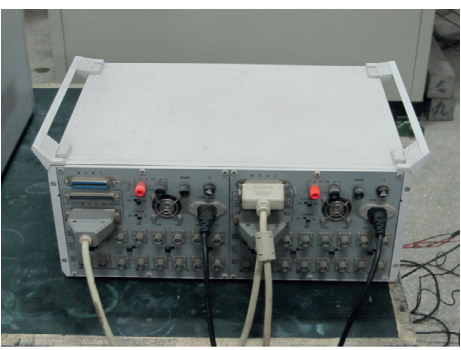

(d)

FIGURE 2: The experimental setup: (a) press machine; (b) pressure sensor; (c) electronic displacement meter; (d) dynamic resistance strain gauge.

For the loading time from the beginning to end, the time for cement particles is shortest, $22 \mathrm{~s}$. For different cement mortar particles, the time for $5 \mathrm{~cm}$ cement mortar particles is the longest, $62 \mathrm{~s}$, the time for other particles is close, about $40-45 \mathrm{~s}$, and the time for 3,4 , and $5 \mathrm{~cm}$ mixed particles is the shortest. In other words, when the material and loading condition are the same, the better the gradation of particle system is, the shorter the time to reach the steady state is.

3.2. Local Load. The local load time-history curves are shown in Figure 6. In the initial stage of loading, the local load of cement mortar particles at the bottom is near zero. The reason is that there is no contact between the particle and the pin which is connected with the pressure sensor. As the particles break and flow, they come into contact with the pin, and the local load begins to rise. The fluctuations occur during the increase of local load, which is the same reason why the overall load has fluctuations. For the cement particle system, there is contact between the particle and the pin in the initial state. With the increase of the applied loading, the local load gradually increases. Although the local load has fluctuations in the loading process, the amplitude of fluctuations is significantly less than that of cement mortar particles.

For the 3,4 , and $5 \mathrm{~cm}$ mixed cement mortar particles under $30 \mathrm{kN}$ loading, the local load time-history curves are obtained from two monitoring points at the bottom of the fixture (see Figure 7). There are great differences between the two curves in 


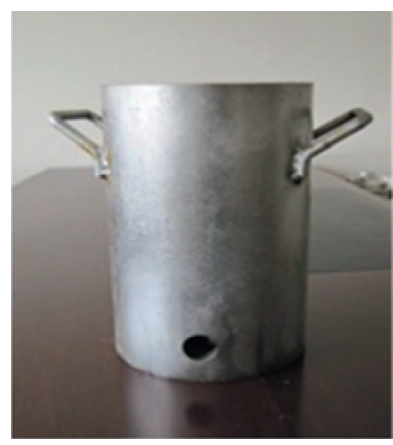

(a)

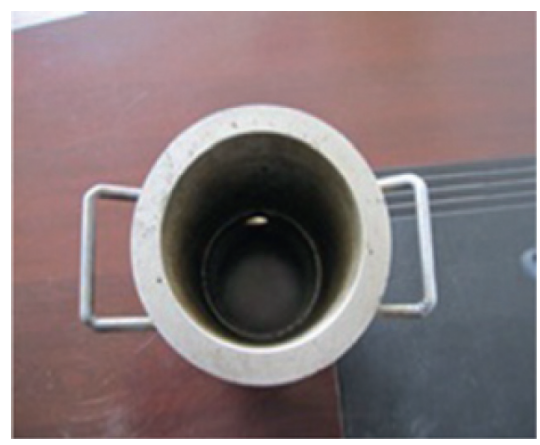

(b)

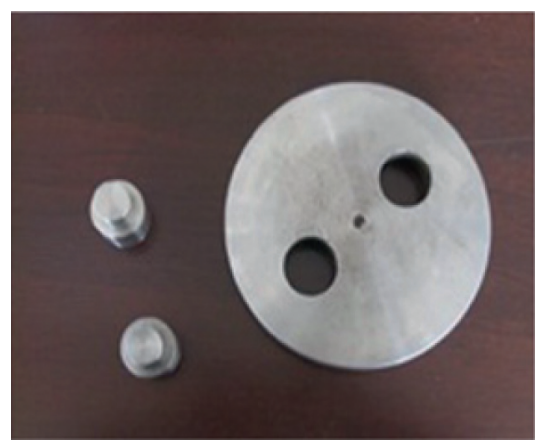

(c)

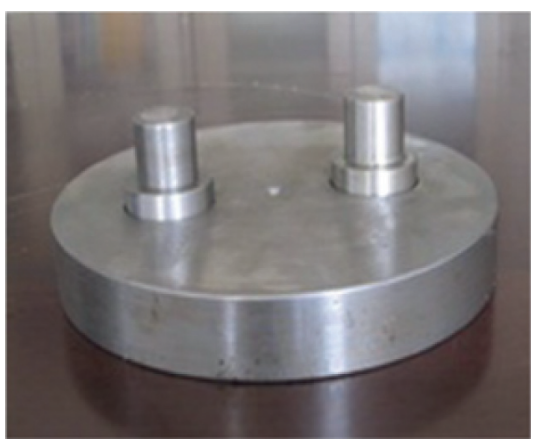

(d)

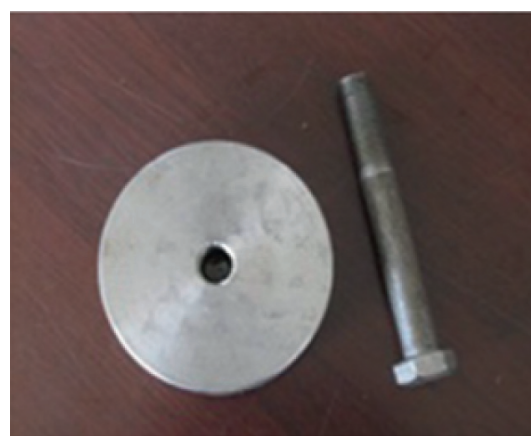

(e)

Figure 3: The container for granular material: $(a, b)$ the fixture; $(c, d)$ lower bearing plate and pins; (e) upper bearing plate.

TABle 1: Experiment groups.

\begin{tabular}{|c|c|c|c|c|}
\hline Experiment group & Particle size $(\mathrm{cm})$ & Particle number & Loading strength $(\mathrm{kN})$ & Experiment no. \\
\hline \multirow{4}{*}{ Cement mortar particle crushing experiments } & \multirow{4}{*}{3} & \multirow{3}{*}{20} & 30 & 1 \\
\hline & & & 40 & 2 \\
\hline & & & 50 & 3 \\
\hline & & \multirow{4}{*}{17} & 30 & 4 \\
\hline \multirow[t]{3}{*}{ Cement mortar particle crushing experiments } & \multirow[t]{3}{*}{4} & & 40 & 5 \\
\hline & & & 50 & 6 \\
\hline & & & 30 & 7 \\
\hline \multirow[t]{3}{*}{ Cement mortar particle crushing experiments } & \multirow[t]{2}{*}{5} & \multirow[t]{2}{*}{12} & 40 & 8 \\
\hline & & & 50 & 9 \\
\hline & 3 & 8 & 30 & 10 \\
\hline \multirow[t]{3}{*}{ Mixed cement mortar particle crushing experiments } & 4 & 8 & 40 & 11 \\
\hline & - & - & 50 & 12 \\
\hline & 4 & 11 & 30 & 13 \\
\hline \multirow[t]{3}{*}{ Mixed cement mortar particle crushing experiments } & 5 & 7 & 40 & 14 \\
\hline & - & - & 50 & 15 \\
\hline & 3 & 10 & 30 & 16 \\
\hline \multirow[t]{3}{*}{ Mixed cement mortar particle crushing experiments } & 4 & 10 & 40 & 17 \\
\hline & 5 & 5 & 50 & 18 \\
\hline & $0.6 \sim 0.8$ & 100 & 30 & 19 \\
\hline \multirow{3}{*}{ Cement particle crushing experiments } & $1.0 \sim 1.2$ & 80 & 40 & 20 \\
\hline & $1.2 \sim 1.6$ & 60 & \multirow{2}{*}{50} & \multirow{2}{*}{21} \\
\hline & $1.6 \sim 2.0$ & 40 & & \\
\hline
\end{tabular}

terms of trend and amplitude due to the differences of the gradation and arrangement of particles. In addition, the flow and rearrangement of the mixed particles during the loading process are random. Therefore, the load at the bottom is not uniformly distributed along the contact points, which is significantly different from the continuous medium.
3.3. Overall Deformation. The overall deformation curve is shown in Figure 8. With the increase of applied load, the overall deformation gradually increases, but the increase rate gradually decreases. The deformable space of the particle system becomes smaller and smaller with the particles breaking and flowing, so the increase rate of overall 


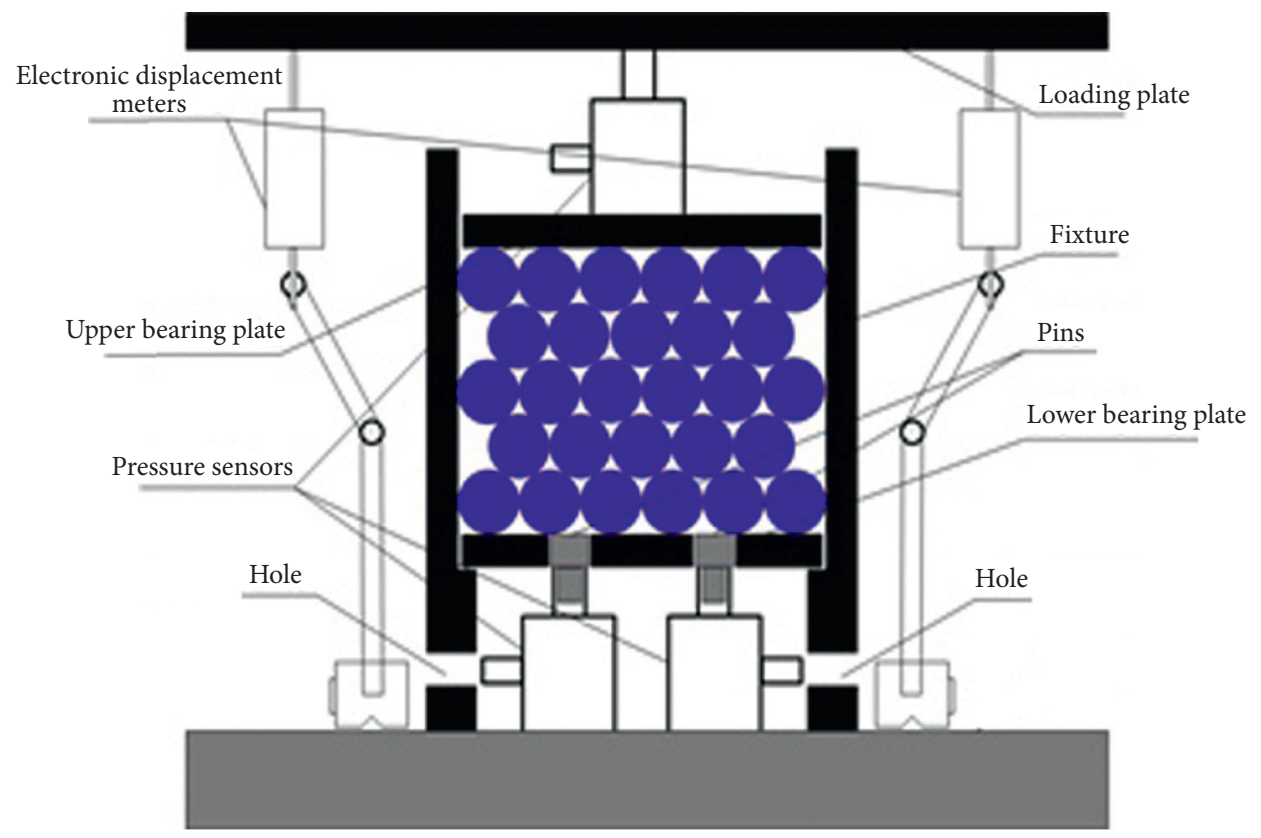

FIgURE 4: Sketch of particle crushing experiment.

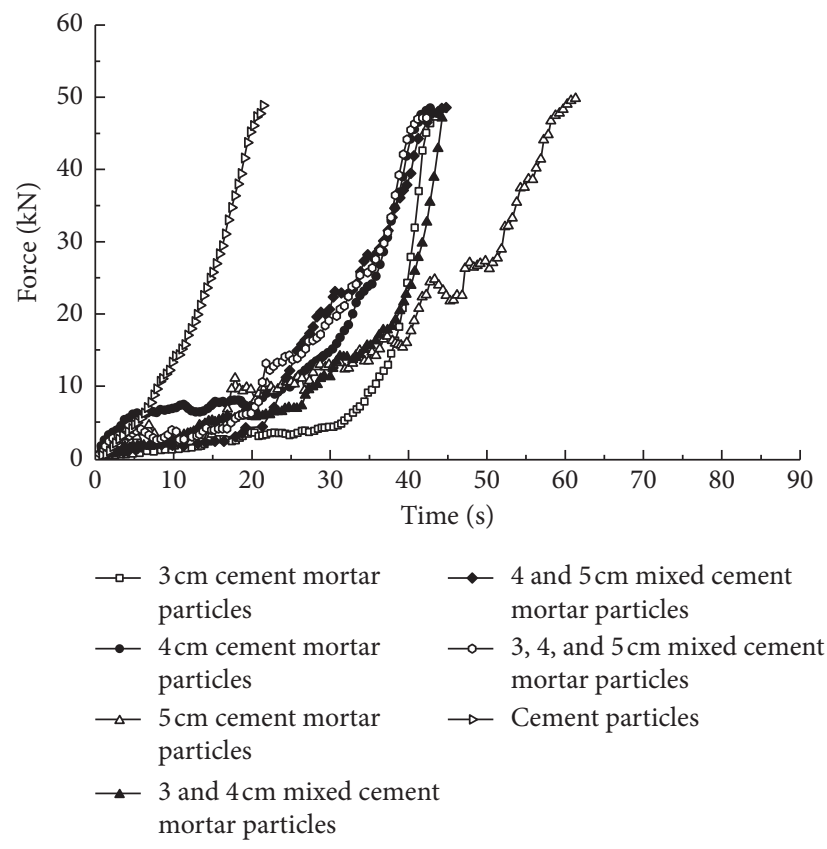

FIgURE 5: The overall load time-history curves of different particles under the same loading.

deformation decreases gradually. Under the same loading, the deformation values of cement mortar particles are obviously higher than those of cement particles. For the cement mortar particles with different gradations, the deformation values for 3, 4, and $5 \mathrm{~cm}$ mixed particles are obviously lower than those for particles with other gradations; this indicates that the higher the compressive strength and the better gradation the particles, the better the ability to resist deformation.

\subsection{Fragment Size Distribution}

3.4.1. Fragment Size Distribution for Same Particles under Different Loading. The breakage of cement particles and 3 and $4 \mathrm{~cm}$ mixed cement mortar particles under the loading of 30,40 , and $50 \mathrm{kN}$ is shown in Figure 9. Under the loading of $30 \mathrm{kN}$, the surface particles of cement particles contact with upper bearing plate, the contact particles have obvious plastic deformation, the particles have cracks, and a small 


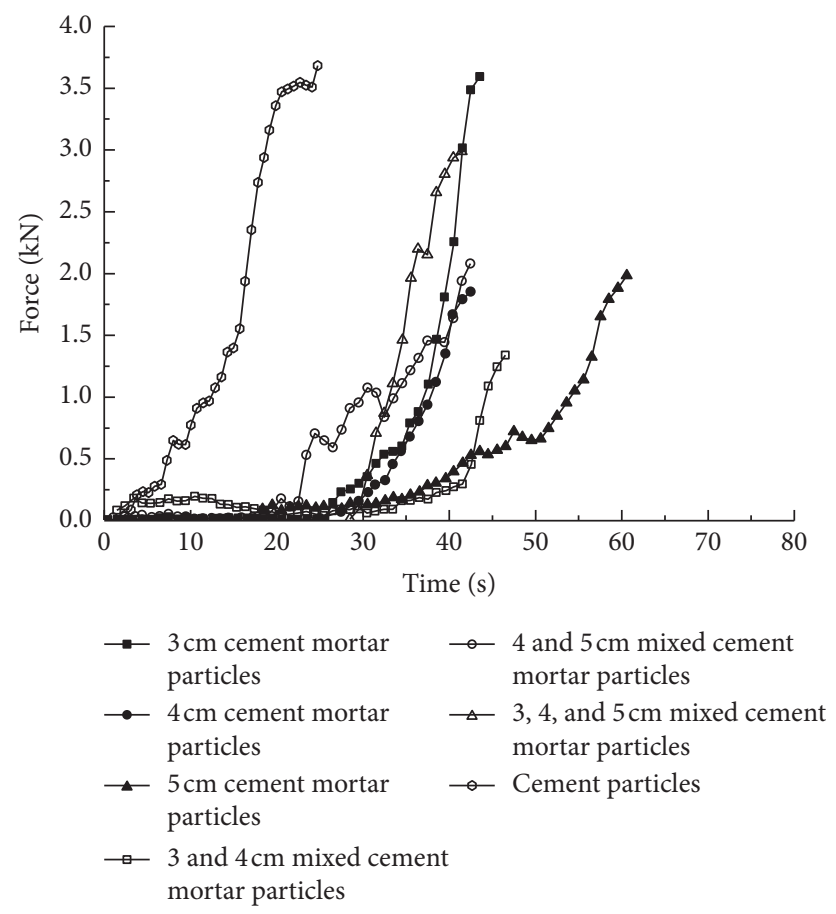

Figure 6: The local load time-history curves of different particles under the same loading.

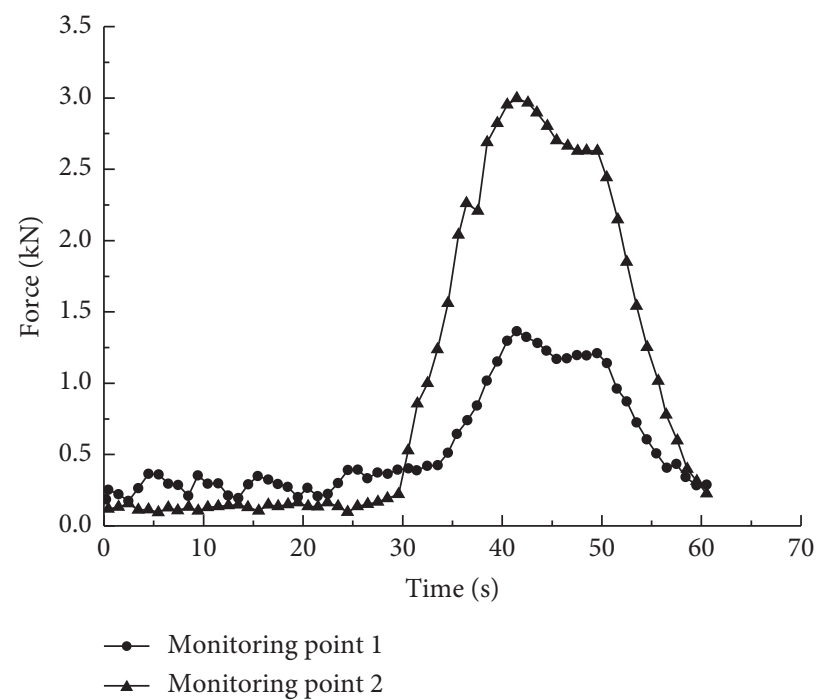

FIgURE 7: The two local load time-history curves in the same experiment.

number of particles are broken. With the increase of loading, the number of broken particles also increases. Under the loading of $30 \mathrm{kN}$, the surface particles of cement mortar particles are seriously damaged, most of the surface particles are broken, and only some surface particles are cracked. When the loading increases to $40 \mathrm{kN}$, the surface particles are all powdered.

The sieving curves of 3 and $4 \mathrm{~cm}$ mixed cement mortar particles and cement particles under the loading of 30,40 , and $50 \mathrm{kN}$ are shown in Figure 10. Under the loading of $30 \mathrm{kN}$, the mass of fragments less than $1 \mathrm{~mm}$ in diameter accounts for $22 \%$ of the total mass, and the mass of fragments less than $10 \mathrm{~mm}$ in diameter accounts for $43 \%$. Under the loading of $50 \mathrm{kN}$, the mass of fragments less than $1 \mathrm{~mm}$ in diameter accounts for $37 \%$ of the total mass, and the mass of fragments less than $10 \mathrm{~mm}$ in diameter accounts for $61 \%$. This indicates that with the increase of loading, the mass proportion of fragments whose diameter is smaller than a 


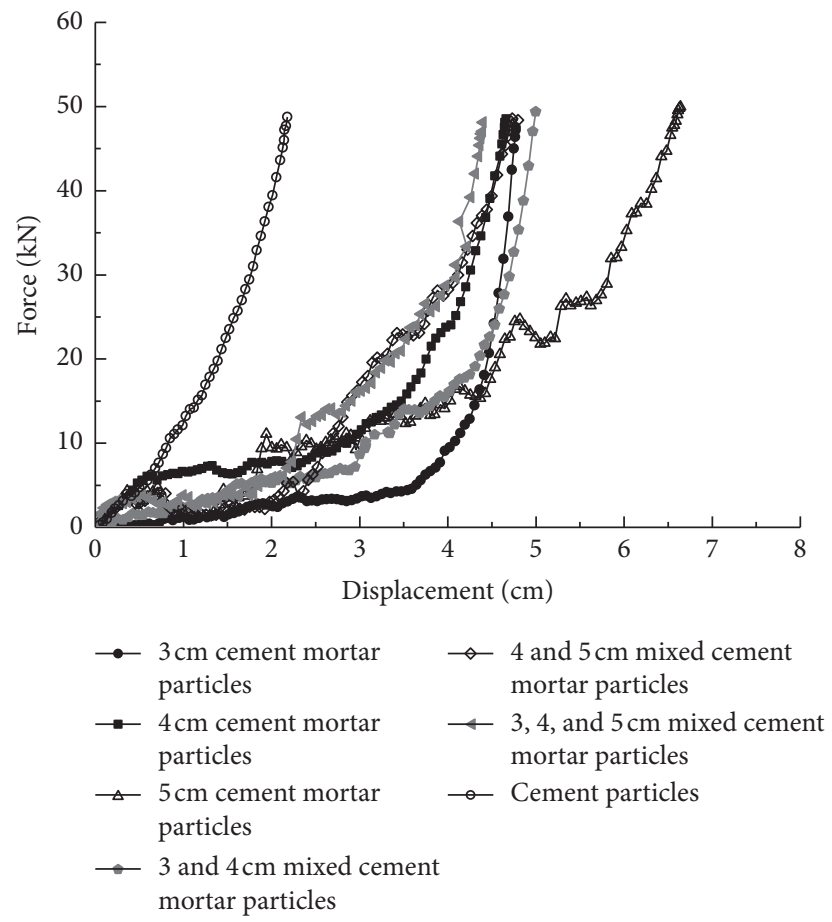

FIgURE 8: The overall deformation curve of different particles under the same loading.

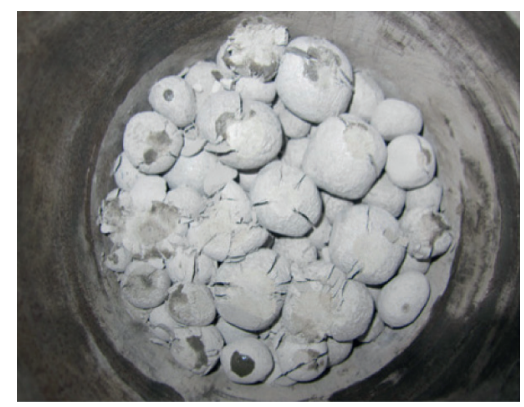

(a)

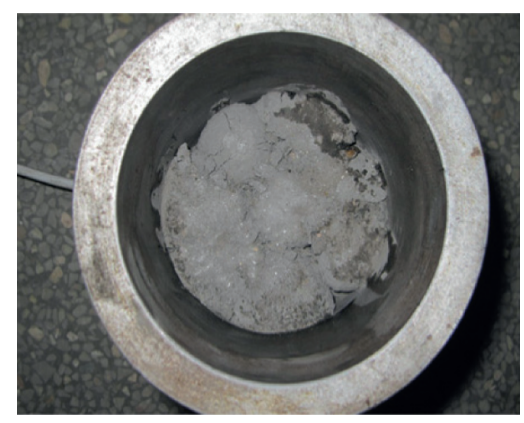

(d)

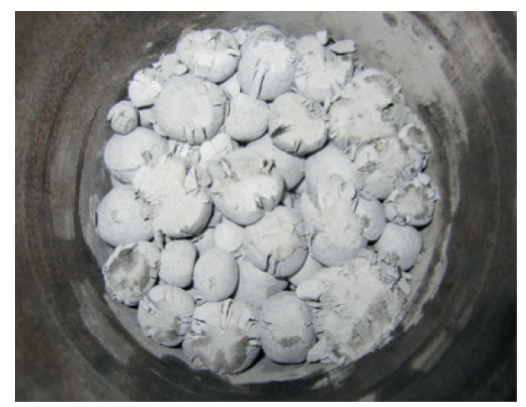

(b)

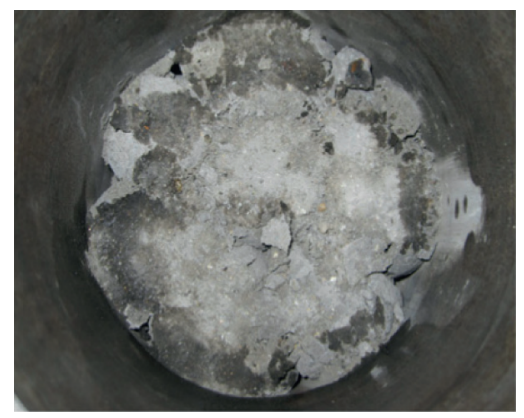

(e)

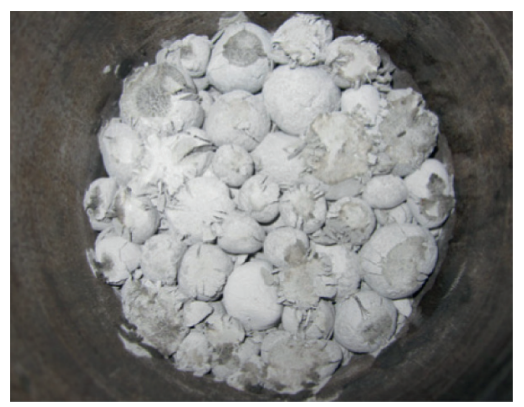

(c)

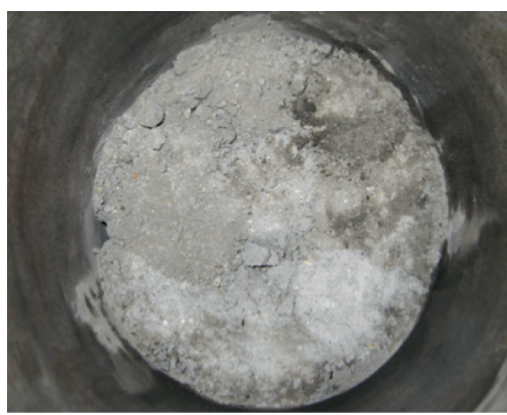

(f)

FIgURE 9: The breakage of particles under the loading of 30,40 , and $50 \mathrm{kN}$ : (a-c) cement particles; (d-f) cement mortar particles.

certain size increases significantly, and the degree of particle crushing increases accordingly. The cement particles also have the same phenomenon.
3.4.2. Fragment Size Distribution for Different Particles under the Same Loading. The sieving curves for different particles under the same loading are shown in Figure 11. The size 


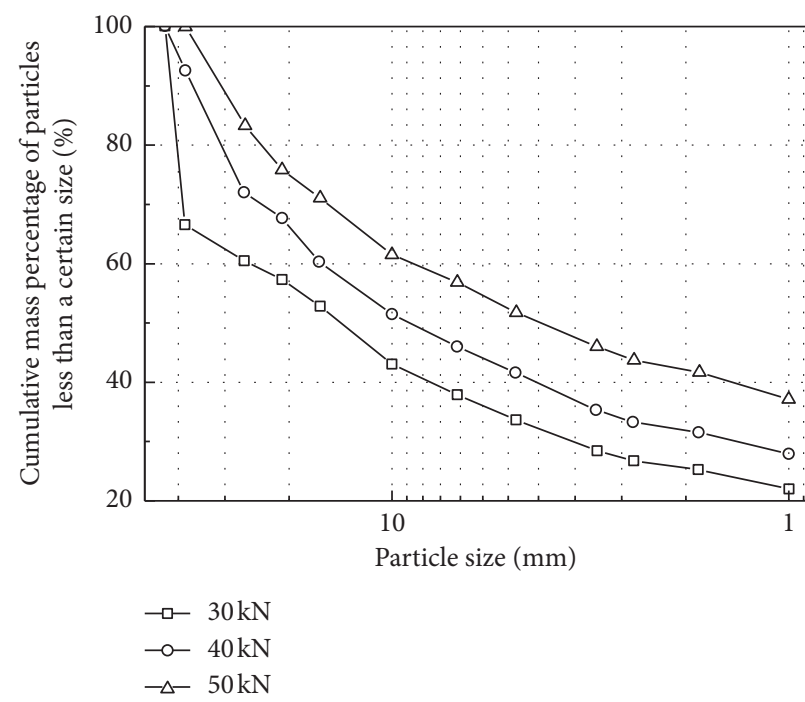

(a)

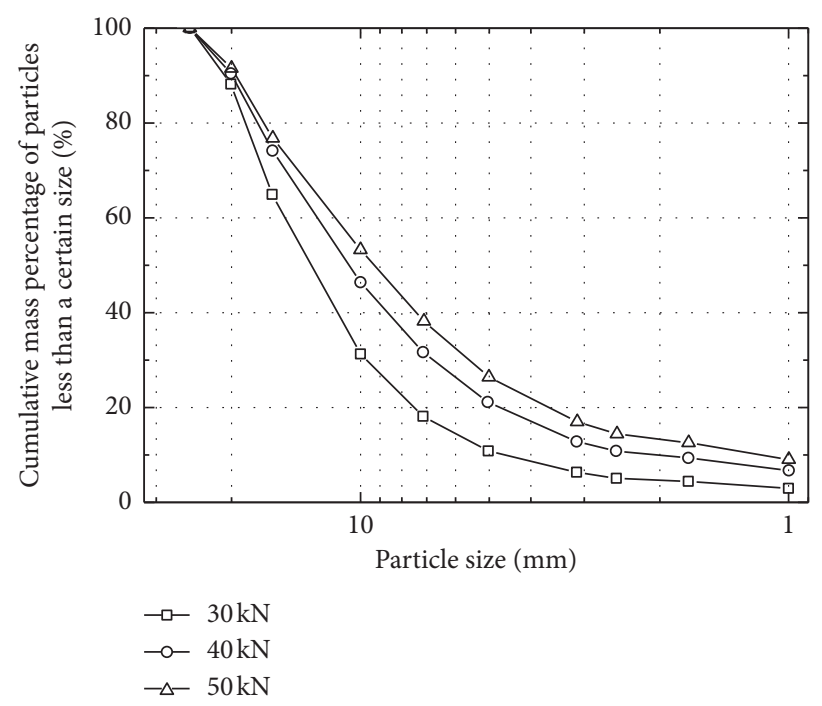

(b)

FIGURE 10: The fragment sieving curves of particles under the loading of 30, 40, and $50 \mathrm{kN}$ : (a) 3 and $4 \mathrm{~cm}$ mixed cement mortar particles; (b) cement particles.

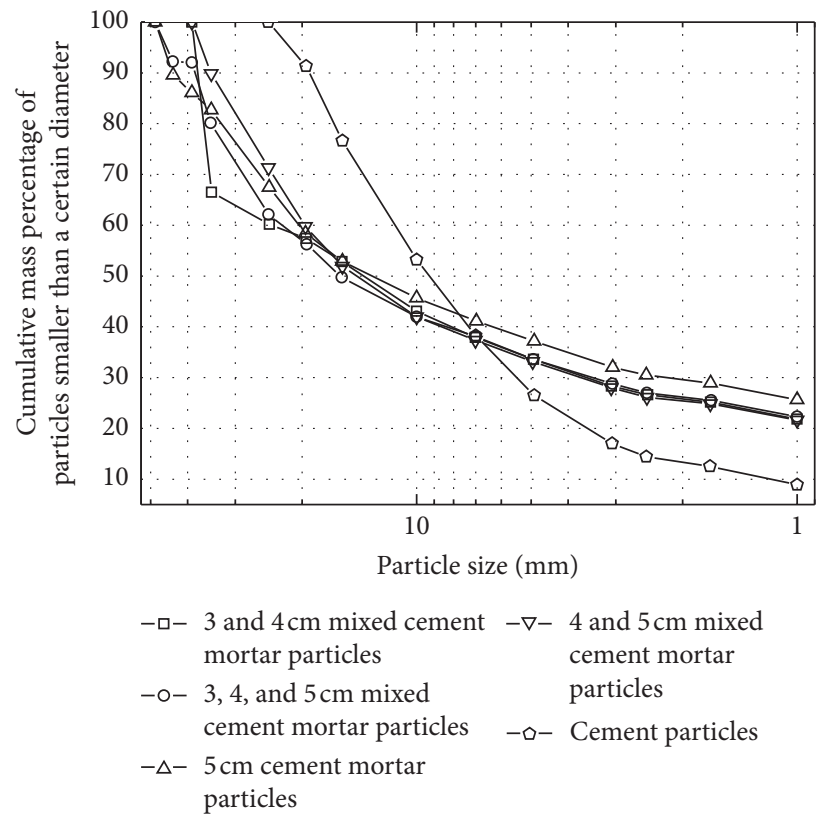

FIgURE 11: The size distribution for different particles under the same loading.

distributions of cement mortar particles with different sizes are relatively close, which shows that in this experimental study, the size and gradation of particles have little influence on particle crushing.

Under the same loading, for the cement particles, the mass of fragments whose diameter is less than $1 \mathrm{~mm}$ accounts for $9 \%$ of the total mass; however, for the cement mortar particles, the mass of fragments whose diameter is less than $1 \mathrm{~mm}$ accounts for $21 \% \sim 25 \%$. This indicates that the cement mortar particles with lower compressive strength are damaged severely, and the proportion of powdery fragments is higher than the proportion for cement particles with higher compressive strength. The compressive strength of particle has a great influence on the fragment size distribution.

3.5. Stratified Failure Characteristic. In this work, the particles are divided into three layers from top to bottom, and the sieving was performed layer by layer to determine the size distribution.

For the 3 and $4 \mathrm{~cm}$ mixed cement mortar particles (see Figure 12), under the loading of $30 \mathrm{kN}$, the particles in the surface layer are damaged seriously, most of them are 


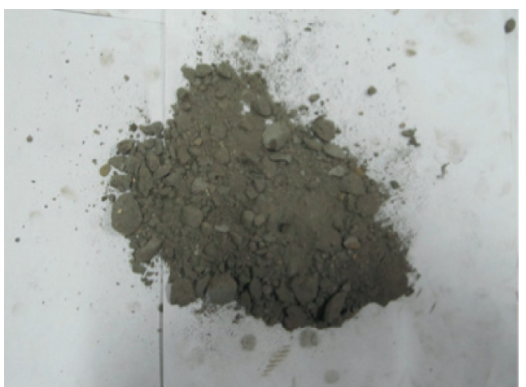

(a)

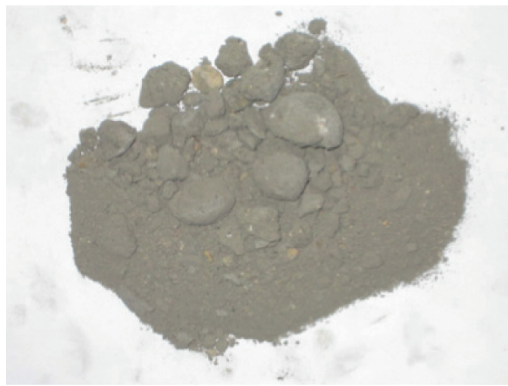

(d)

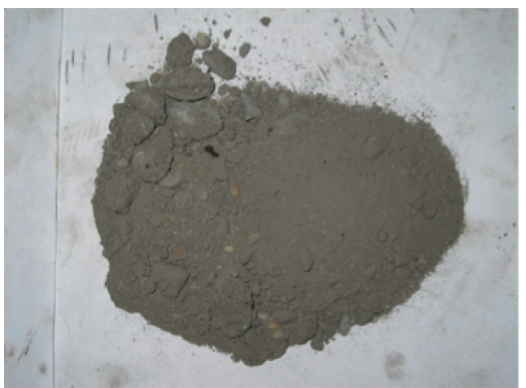

(g)

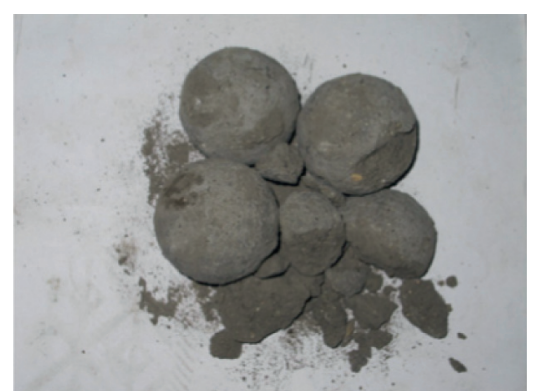

(b)

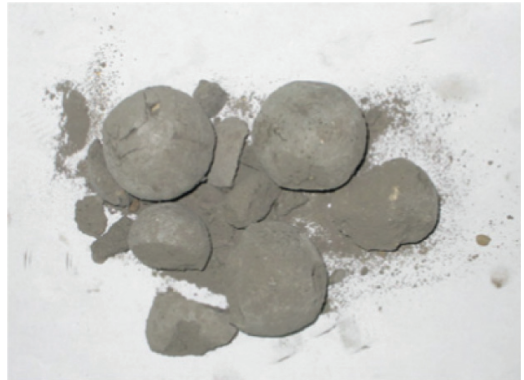

(e)

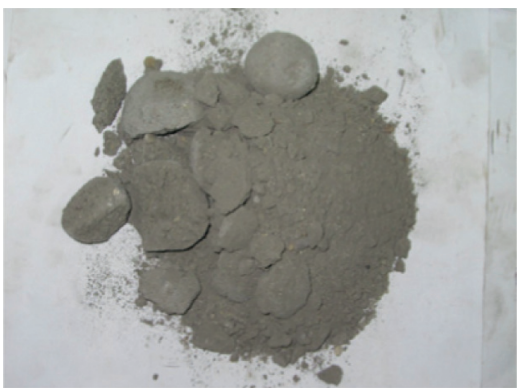

(h)

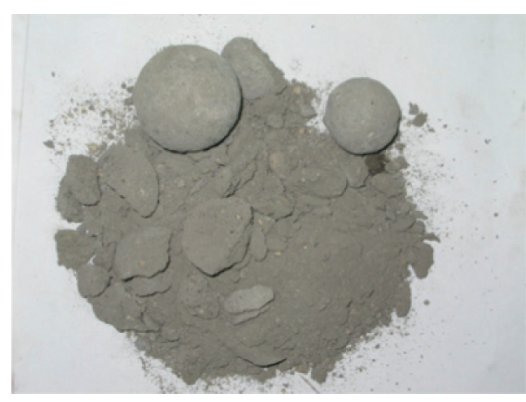

(c)

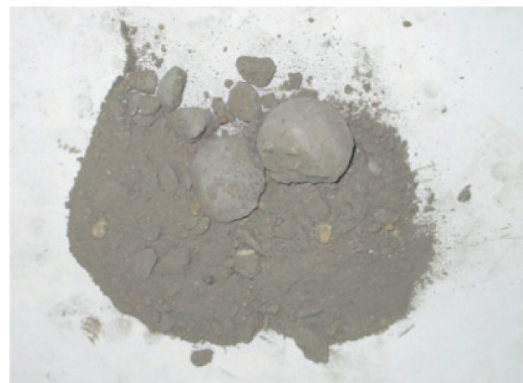

(f)

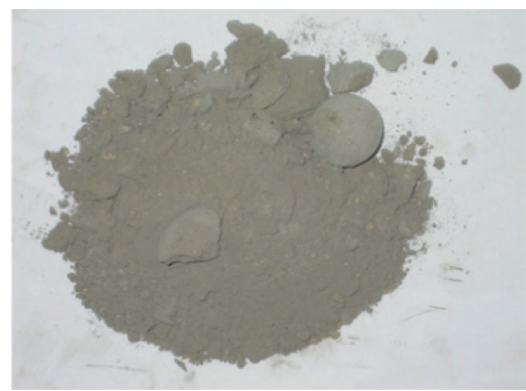

(i)

Figure 12: The breakage of 3 and $4 \mathrm{~cm}$ mixed cement mortar particles under the loading of 30,40 , and $50 \mathrm{kN}$ : (a-c) in the surface layer; (d-f) in the medium layer; ( $\mathrm{g}-\mathrm{i})$ in the bottom layer.

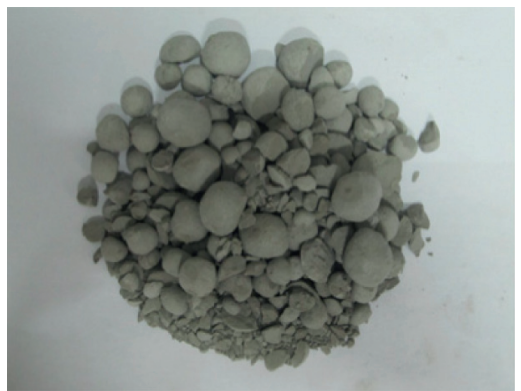

(a)

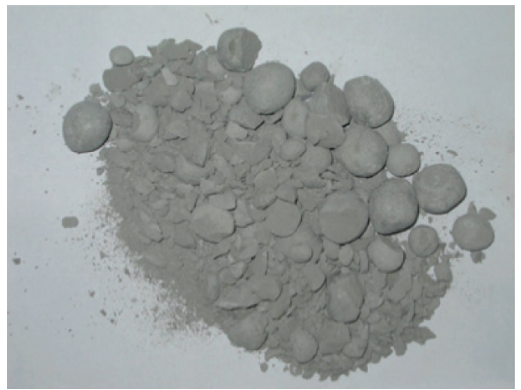

(d)

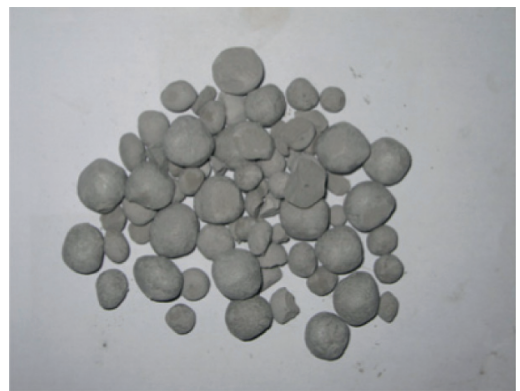

(b)

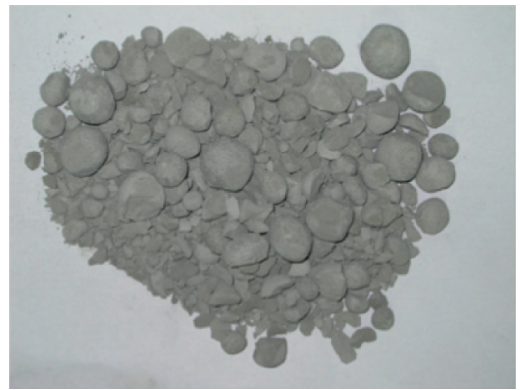

(e)

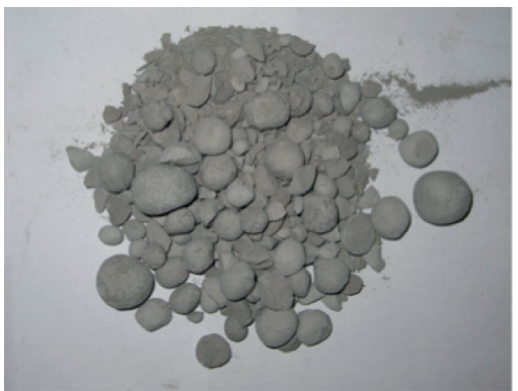

(c)

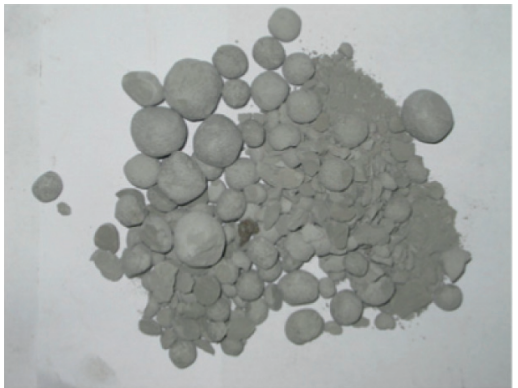

(f)

Figure 13: Continued. 


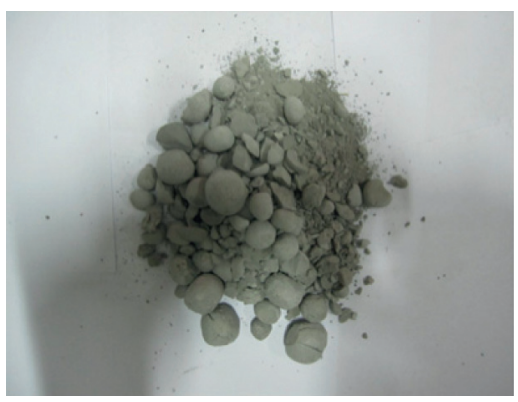

$(\mathrm{g})$

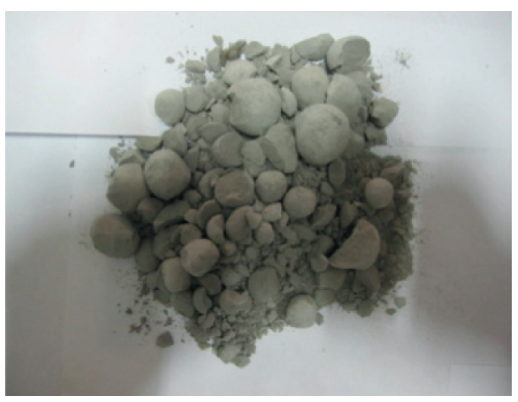

(h)

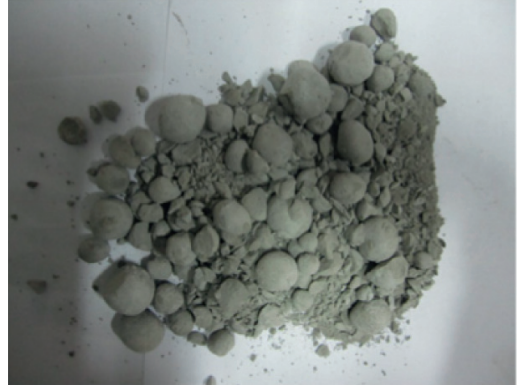

(i)

Figure 13: The breakage of cement particles under the loading of 30,40 , and $50 \mathrm{kN}$ : $(\mathrm{a}-\mathrm{c})$ in the surface layer; (d-f) in the medium layer; $(\mathrm{g}-\mathrm{i})$ in the bottom layer.

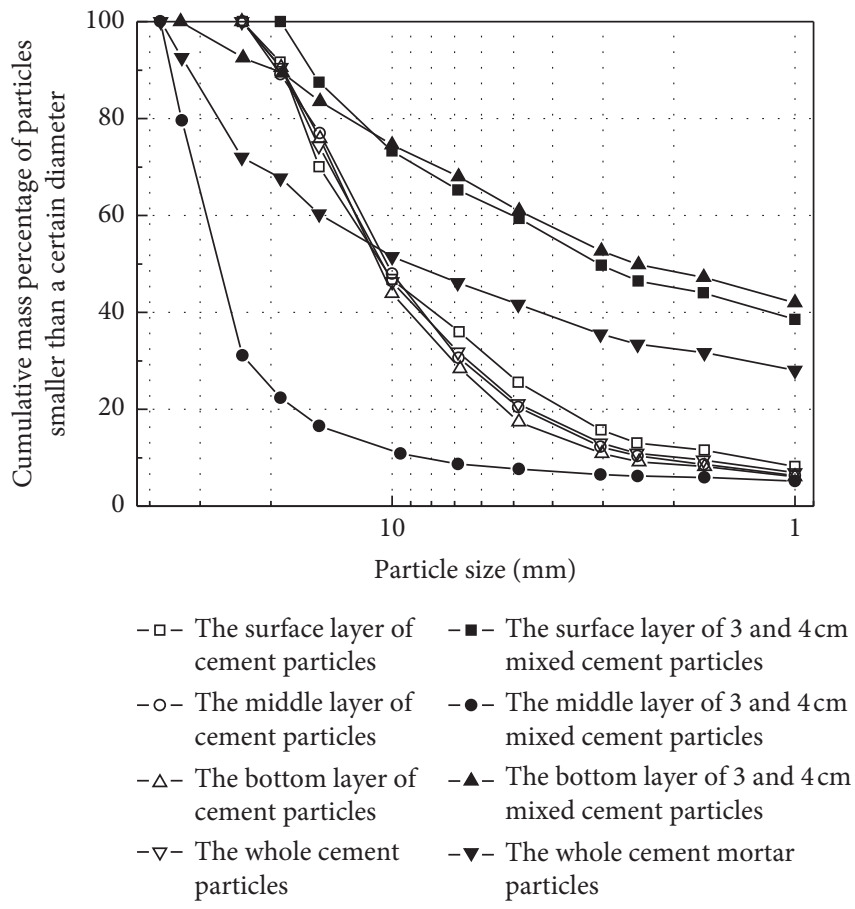

(a)

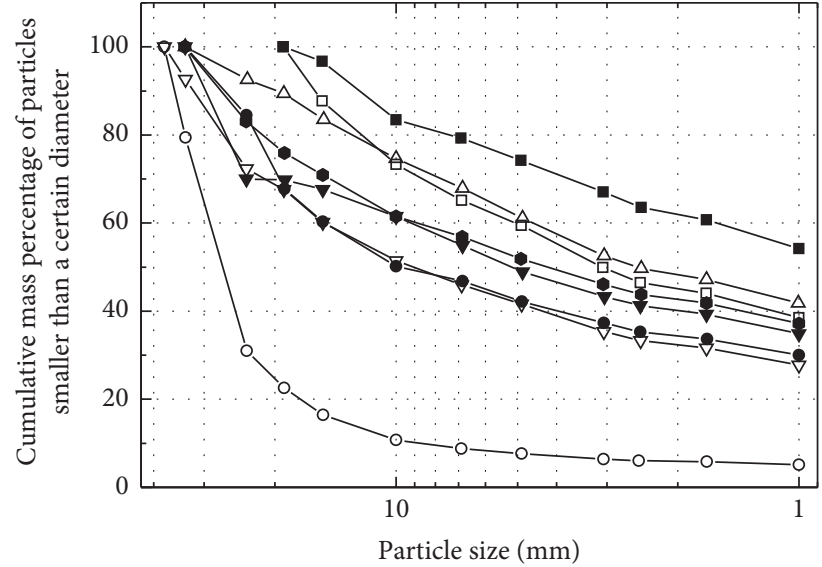

- - The surface layer particles - - - - The surface particles under $30 \mathrm{kN}$

- ०- The middle layer particles - $\bullet$ - The middle particles under $30 \mathrm{kN}$ under $40 \mathrm{kN}$

$-\Delta_{-}$The bottom layer particles - $\mathbf{\nabla}-$ The bottom particles under $30 \mathrm{kN} \quad$ under $40 \mathrm{kN}$

$-\nabla-$ The whole particles $\quad-\quad$ The whole particles under $30 \mathrm{kN} \quad$ under $40 \mathrm{kN}$

(b)

FIgURE 14: The stratified sieving curves: (a) 3 and $4 \mathrm{~cm}$ mixed cement mortar and cement particles under the loading of $30 \mathrm{kN}$; (b) 3 and $4 \mathrm{~cm}$ mixed cement mortar particles under the loading of 30 and $40 \mathrm{kN}$.

powdered, and the rest are broken into small pieces. In the bottom layer, some of the particles are broken into large pieces, and some are only cracked and not broken. The particles in the medium layer are damaged lighter than the particles in the above layers, and most of them are only cracked. When the applied load increases, the degree of damage also increases. For the cement particles (see Figure 13), the damage of different layers is similar, and no obvious stratified damage occurs.

The stratified sieving curves of 3 and $4 \mathrm{~cm}$ mixed cement mortar particles and cement particles under the loading of
$30 \mathrm{kN}$ are shown in Figure 14(a). For the mixed cement mortar particles, the curves have distinct differences, the mass of particles in surface and bottom layers whose diameter is less than $1 \mathrm{~mm}$ accounts for $40 \%$ of the total mass, and the mass whose diameter is less than $10 \mathrm{~mm}$ accounts for $72 \%$; the maximum size of particles in the surface layer is less than $20 \mathrm{~mm}$. The mass of particles in medium layers whose diameter is less than $1 \mathrm{~mm}$ accounts for $5 \%$ of the total mass, and the mass whose diameter is less than $10 \mathrm{~mm}$ accounts for $10 \%$; the maximum size of particles in the surface layer is close to $40 \mathrm{~mm}$. For the cement particles, the curves are very 
similar. From the above, it can be seen that the cement mortar particles with lower strength have obvious stratified failure characteristic.

The stratified sieving curves of 3 and $4 \mathrm{~cm}$ mixed cement mortar particles under the loading of 30 and $40 \mathrm{kN}$ are shown in Figure 14(b). Under the loading of $40 \mathrm{kN}$, the curves gaps are small; as the applied load increases, the degree of particle crushing in the medium layer increases, and the stratified failure characteristic decreases.

\section{Conclusions}

In this paper, a systematic method was proposed to measure the mechanical behavior of granular material, and the experiments were performed with different sizes and gradation particles. The overall load, local load, overall displacement, and fragment size distribution were analyzed and compared, and the main conclusions are shown below:

(1) During the loading, the fracture and flow of the particles lead to repeated loading-unloadingreloading phenomenon, and the overall load rises in a fluctuating manner. When all the voids are filled, the overall load increases to the end at a steady rate.

(2) During the loading, as the force chain with different strength is formed inside the granular particles, the force distribution in the bottom of granular particles is not uniform according to the contact points, which is significantly different from the load distribution in the continuous medium method.

(3) During the loading, the increase rate of the overall displacement gradually decreases. The magnitude of the displacement depends on the compressive strength and gradation of particles.

(4) The cement mortar particles have obvious stratified failure characteristic in the low loading, and as the loading increases, the stratified failure characteristic will be weakened. For the cement particles whose compressive strength is higher than cement mortar particles, there was no obvious stratified failure characteristic. So, the compressive strength and loading strength have a great influence on the fragment size distribution of particles.

\section{Data Availability}

The data used to support the findings of this study are available from the corresponding author upon request.

\section{Conflicts of Interest}

The authors declare that they have no conflicts of interest.

\section{Authors' Contributions}

Futian Zhao analyzed the data and wrote the paper. Jun Liu was responsible for data. Zhimin Xiao, Mingqing Liu, Yue
Wang, Chen Ou, and Mengyang Zhen contributed to experimental testing.

\section{Acknowledgments}

This study was supported by the National Natural Science Foundation of China (nos. 51874118 and 51778211), the Fundamental Research Funds for the Central Universities (no. KYCX18-0567), and the Jiangsu Provincial Natural Science Foundation of China (no. BK20171434).

\section{References}

[1] P. G. Gennes de, "Reflections on the mechanics of granular matter," Physica A: Statistical Mechanics and its Applications, vol. 261, no. 3-4, pp. 267-293, 1998.

[2] P. G. Genres de, "Granular matter: a tentative view," Reviews of Modern Physics, vol. 71, pp. 5374-S382, 1999.

[3] B. O. Hardin, "Crushing of soil particles," Journal of Geotechnical Engineering, vol. 111, no. 10, pp. 1177-1192, 1985.

[4] P. V. Lade, J. A. Yamamuro, and P. A. Bopp, "Significance of particle crushing in granular materials," Journal of Geotechnical Engineering, vol. 122, no. 4, pp. 309-316, 1996.

[5] K. L. Lee and I. Farhoomand, "Compressibility and crushing of granular soil in anisotropic triaxial compression," Canadian Geotechnical Journal, vol. 4, no. 1, pp. 68-86, 1967.

[6] R. Pitchumani, N. Gupta, G. M. H. Meesters, and B. Scarlett, "Analysis of single particle attrition during impact experiments," Particle \& Particle Systems Characterization, vol. 20, no. 5, pp. 323-326, 2003.

[7] P. J. Hill, "Statistics of multiple particle breakage accounting for particle shape," AIChE Journal, vol. 50, no. 5, pp. 937-952, 2004.

[8] I. Einav, "Breakage mechanics-part I: theory," Journal of the Mechanics and Physics of Solids, vol. 55, no. 6, pp. 1274-1297, 2007.

[9] I. Einav, "Breakage mechanics-part II: modelling granular materials," Journal of the Mechanics and Physics of Solids, vol. 55, no. 6, pp. 1298-1320, 2007.

[10] J. P. Hyslip and L. E. Vallejo, "Fractal analysis of the roughness and size distribution of granular materials," Engineering Geology, vol. 48, no. 3-4, pp. 231-244, 1997.

[11] D. Markauskas and R. Kačianauskas, "Compacting of particles for biaxial compression test by the discrete element method," Journal of Civil Engineering and Management, vol. 12, no. 2, pp. 153-161, 2006.

[12] G. R. McDowel, "The role of particle crushing in granular materials," Modern Trends in Geomechanics, vol. 106, pp. 271-288, 2006.

[13] H. Arslan, G. Baykal, and S. Sture, "Analysis of the influence of crushing on the behavior of granular materials under shear," Granular Matter, vol. 11, no. 2, pp. 87-97, 2009.

[14] J. Feda, "Notes on the effect of grain crushing on granular soil behavior," Engineering Geology, vol. 63, pp. 93-98, 2002.

[15] R. P. Jensen, M. E. Plesha, T. B. Edil, P. J. Bosscher, and N. B. Kahla, "DEM simulation of particle damage in granular media-structure interfaces," International Journal of Geomechanics, vol. 1, no. 1, pp. 21-39, 2001.

[16] C. Silvani, T. Désoyer, and S. Bonelli, "Discrete modelling of time-dependent rockfill behaviour," International Journal for Numerical and Analytical Methods in Geomechanics, vol. 33, no. 5, pp. 665-685, 2008. 
[17] G. Marketos and M. D. Bolton, "Compaction bands as observed in DEM simulations," Powder and Grains, vol. 2, pp. 1405-1409, 2005.

[18] A. Mohamed and M. Gutierrez, "Comprehensive study of the effects of rolling resistance on the stress-strain and strain localization behavior of granular materials," Granular Matter, vol. 12 , no. 5, pp. 527-541, 2010.

[19] J. Tejchman, "Effect of boundary, shear rate and grain crushing on shear localization in granular materials within micro-polar hypoplasticity," Advanced Structured Materials, vol. 1, pp. 363-376, 2010.

[20] D. Robertson, M. D. Bolton, and G. R. McDowell, "A numerical representation of fracturing granular materials," International Journal for Numerical and Analytical Methods in Geomechanics, vol. 21, no. 12, pp. 825-843, 1997.

[21] D. A. Gorham and A. D. Salman, "The failure of spherical particles under impact," Wear, vol. 258, no. 1-4, pp. 580-587, 2005.

[22] J. Fu, G. K. Reynolds, M. J. Adams, M. J. Hounslow, and A. D. Salman, "An experimental study of the impact breakage of wet granules," Chemical Engineering Science, vol. 60, no. 14, pp. 4005-4018, 2005.

[23] S. Antonyuk, J. Tomas, S. Heinrich, and L. Mörl, "Breakage behaviour of spherical granulates by compression," Chemical Engineering Science, vol. 60, no. 14, pp. 4031-4044, 2005.

[24] S. Antonyuk, S. Heinrich, J. Tomas, N. G. Deen, M. S. Van Buijtenen, and J. A. M. Kuipers, "Energy absorption during compression and impact of dry elastic-plastic spherical granules," Granular Matter, vol. 12, no. 1, pp. 15-47, 2010.

[25] S. Antonyuk, M. Khanal, J. Tomas, S. Heinrich, and L. Mörl, "Impact breakage of spherical granules: experimental study and DEM simulation," Chemical Engineering and Processing: Process Intensification, vol. 45, no. 10, pp. 838-856, 2006.

[26] K. T. Chau, X. X. Wei, R. H. C. Wong, and T. X. Yu, "Fragmentation of brittle spheres under static and dynamic compressions: experiments and analyses," Mechanics of Materials, vol. 32, no. 9, pp. 543-554, 2000.

[27] W. Schubert, M. Khanal, and J. Tomas, "Impact crushing of particle-particle compounds- experiment and simulation," International Journal of Mineral Processing, vol. 75, no. 1, pp. 41-52, 2005.

[28] J. A. Åström and H. J. Herrmann, "Fragmentation of grains in a two-dimensional packing," The European Physical Journal B, vol. 5, no. 3, pp. 551-554, 1998.

[29] T. Olivier, V. Denis, and C. Jean-Claude, "Sizes effects in single grain fragmentation," Granular Matter, vol. 2, pp. 1927, 1999.

[30] U. Jansen and D. Stoyan, "On the validity of the Weibull failure model for brittle particles," Granular Matter, vol. 2, no. 4, pp. 165-170, 2000.

[31] G. R. Mcdowell and M. D. Bolton, "On the micromechanics of crushable aggregates," Géotechnique, vol. 48, no. 5, pp. 667-679, 1998 .

[32] P. V. Lade and J. A. Yamamuro, "Undrained sand behavior in axisymmetric tests at high pressures," Journal of Geotechnical Engineering, vol. 122, no. 2, pp. 120-129, 1996.

[33] J. A. Yamamuro and P. V. Lade, "Drained sand behavior in axisymmetric tests at high pressures," Journal of Geotechnical Engineering, vol. 122, no. 2, pp. 109-119, 1996.

[34] L. E. Vallejo, S. Lobo-Guerrero, and Z. Chik, "A network of fractal force chains and their effect in granular materials under compression," Fractals in Engineering, vol. 2, pp. 67-80, 2005.

[35] T. G. Sitharam and M. S. Nimbkar, "Micromechanical modeling of granular materials: effect of particle size and gradation," Geotechnical and Geological Engineering, vol. 18, no. 2, pp. 91-117, 2000.

[36] K. Grine, A. Attar, A. Aoubed, and D. Breysse, "Using the design of experiment to model the effect of silica sand and cement on crushing properties of carbonate sand," Materials and Structures, vol. 44, no. 1, pp. 195-203, 2011.

[37] M. M. Hagerty, D. R. Hite, C. R. Ullrich, and D. J. Hagerty, "One-Dimensional high-pressure compression of granular media," Journal of Geotechnical Engineering, vol. 119, no. 1, pp. 1-18, 1993.

[38] C. Le Bouteiller and M. Naaim, "Aggregate breakage under dynamic loading," Granular Matter, vol. 13, no. 4, pp. 385-393, 2011.

[39] Y. P. Cheng, Y. Nakata, and M. D. Bolton, "Discrete element simulation of crushable soil," Géotechnique, vol. 53, no. 7, pp. 633-641, 2003.

[40] G. Marketos and M. D. Bolton, "Compaction bands simulated in discrete element models," Journal of Structural Geology, vol. 31, no. 5, pp. 479-490, 2009.

[41] C. Nouguier-Lehon, "Effect of the grain elongation on the behaviour of granular materials in biaxial compression," Comptes Rendus Mécanique, vol. 338, no. 10-11, pp. 587-595, 2010.

[42] J. F. Wang and H. B. Yan, "3D DEM simulation of crushable granular soils under plane strain compression condition," Procedia Engineering, vol. 14, pp. 1713-1720, 2011.

[43] Z. Mahmood and K. Iwashita, "Influence of inherent anisotropy on mechanical behavior of granular materials based on DEM simulations," International Journal for Numerical and Analytical Methods in Geomechanics, vol. 34, no. 8, pp. 795-819, 2010.

[44] C. Salot, P. Gotteland, and P. Villard, "Influence of relative density on granular materials behavior: DEM simulations of triaxial tests," Granular Matter, vol. 11, no. 4, pp. 221-236, 2009.

[45] Z. Bi, Q. Sun, F. Jin, and M. Zhang, "Numerical study on energy transformation in granular matter under biaxial compression," Granular Matter, vol. 13, no. 4, pp. 503-510, 2011.

[46] H. Lee, H. Cho, and J. Kwon, "Using the discrete element method to analyze the breakage rate in a centrifugal/vibration mill," Powder Technology, vol. 198, no. 3, pp. 364-372, 2010. 